Check for updates

Cite this: RSC Adv., 2017, 7, 48245

Received 29th June 2017

Accepted 8th October 2017

DOI: 10.1039/c7ra07210d

rsc.li/rsc-advances

\title{
2-SeCD treatment extends lifespan, improves healthspan and enhances resistance to stress in Caenorhabditis elegans $\uparrow$
}

\author{
Lin Zhou, (D) ${ }^{\text {ab }}$ Xueqi Fu, (D) ab Yi Luo, (D) ${ }^{c}$ Fangzhou Du, (D) ${ }^{a}$ Hua Wang, (D) \\ Shu Xing, (iD ab Wannan $\mathrm{Li}$ (iD) ab and Junfeng Ma (iD *ab
}

\begin{abstract}
Aging is primarily caused by reactive oxygen species (ROS). 2 -Selenium-bridged $\beta$-cyclodextrin (2-SeCD) is an enzyme with glutathione peroxidase (GPX)-like activity, which indicates that it might be an important ROS scavenger in vivo. However, its effects on aging are unknown. In this study, we studied the dosedependent effects of 2-SeCD on the lifespan, healthspan and resistance to stress of Caenorhabditis elegans. The mean lifespan of nematodes treated with $0.05,0.5$, and $5 \mathrm{mM} 2-\mathrm{SeCD}$ increased significantly in a dose-dependent manner. Healthspan also improved, as revealed by increased motility and GPX activity and decreased lipid peroxidation and ROS level, indicating that DNA damage was curbed. In addition, nematodes treated with 2-SeCD were more resistant to heat stress, UV irradiation and $\mathrm{H}_{2} \mathrm{O}_{2}$-induced oxidative stress. Our findings indicate that 2-SeCD extends the lifespan of $C$. elegans, improves the healthspan and enhances the resistance to stress.
\end{abstract}

\section{Introduction}

Age is a major risk factor for many chronic diseases. In the mid1950s, Denham Harman articulated the free radical theory of aging, speculating that endogenous oxygen radicals, which are generated in cells, cause cumulative damage. ${ }^{1}$ One of the major causes of aging is the generation of reactive oxygen species (ROS); ${ }^{2}$ thus, any factor that can reduce the generation of ROS may delay senescence. During aerobic respiration, organisms produce ROS when electrons are transferred along the respiratory chain to generate ATP. In a normal system, oxygen is the final electron acceptor, thereby yielding water. However, an inappropriate electron donation, usually involving ubiquinone within complex III, generates ROS. ${ }^{3}$ Specifically, ROS are chemical species generated endogenously and exogenously from several different sources, including superoxide anions, hydroxyl radicals and hydrogen peroxide. Several species, such as superoxide anions or hydroxyl radicals, are extremely unstable, whereas others, such as hydrogen peroxide, are freely diffusible and relatively long-lived. Moreover, ROS cause lipid peroxidation, protein oxidation and DNA damage, which are

\footnotetext{
${ }^{a}$ National Engineering Laboratory for AIDS Vaccine, School of Life Sciences, Jilin University, Changchun 130012, China. E-mail: mjf@jlu.edu.cn

${ }^{b}$ Key Laboratory for Molecular Enzymology and Engineering, The Ministry of Education, School of Life Sciences, Jilin University, Changchun 130012, China

'Department of Physiology, University of Texas Southwestern Medical Center, Dallas, TX, USA

$\dagger$ Electronic supplementary information (ESI) available. See DOI: $10.1039 / \mathrm{c} 7 \mathrm{ra} 07210 \mathrm{~d}$
}

deleterious to cells. A rise in intracellular ROS levels can damage various cell components and trigger specific signaling pathways. Both of these effects can influence numerous cellular processes linked to aging and the development of age-related diseases. ${ }^{4}$ It has been shown that treatments that increase oxidative stress promote cellular damage and reduce the lifespan of various species. ${ }^{5}$ ROS-induced damage can shorten the lifespan of organisms by a mechanism that is different from normal aging. Reducing intracellular ROS may, in principle, be achieved by increasing antioxidant defenses in the cell, either by dietary supplements of antioxidants or by overexpression of genes encoding antioxidant enzymes. ${ }^{6}$

Selenium is a necessary trace element of the human body, and it plays an important role in human metabolism. A lack or excess of selenium can cause many kinds of diseases. Glutathione peroxidase (GPX) in all eukaryotic cells is the first enzyme of the selenium family. ${ }^{7}$ It removes hydrogen peroxide and lipid peroxide, which boosts the metabolism of hydrogen peroxide and protects living organisms from oxidative damage. Many diseases, such as Alzheimer's disease, immunological diseases, irradiation damage, and their pathogenesis are associated with changes in antioxidant enzyme levels. ${ }^{8}$ Most GPX studies focus on its catalytic mechanism and active site. Studies on the artificial simulation can not only help to develop viable drugs, but also help to improve the understanding of GPX structure and catalytic function. Cyclodextrin selenium generated (2-SeCD), known as a GPX simulator, is a synthetic organic compound selenide. ${ }^{9}$ It has unique GPX activity, antioxidant and anti-inflammatory properties, with very low toxicity. ${ }^{10}$ It also has a hydrophobic cavity, which is similar to the base enzyme 
activity site, which can wrap the substrate, and the end of the hydroxyl can produce a catalytic site. According to a previous report, 2-SeCD is a very promising compound. It can replace ebselen, known as the GPX simulation. Furthermore, it has enhanced enzyme activity and is water-soluble, ${ }^{11}$ so it can bind a variety of organic molecules and remove ROS. Recently, GPX has been confirmed to show excessive expression in cancer cells and to antitumor effect. ${ }^{12}$ These results suggest that 2-SeCD may be the target of cancer treatment.

Organisms are vulnerable to environmental challenges. With time, the body will deteriorate, increasing the risks of disease and death. Nematodes are good models of aging that are similar with higher mammals, including humans. As they age, they become less fertile, move more slowly and develop sarcoma. They also accumulate oxidants and liposomes, which are common features of aging in many types of. ${ }^{13}$ At the nematode and human levels, genes and proteins involved in aging are $80 \%$ similar. ${ }^{14}$ In addition to biological aging models, nematodes have unique advantages. They are easily cultured and have a short life cycle, allowing rapid replication of experimental treatments. In addition, this model has been successfully applied to pharmacological studies of drugs and interactions between genes. More importantly, vitamins, clinical drugs, antioxidant supplements, and phytochemicals can extend human life and physiological senescence.

Specifically, our objectives were to determine the effects of 2SeCD on the lifespan, healthspan and stress resistance in vivo using C. elegans as a model.

\section{Materials and methods}

\subsection{Chemicals and reagents}

Sodium azide, thiobarbituric acid, glutathione reductase and NADPH were purchased from TransGen Biotech Co., Ltd. (Beijing, China). All other chemicals were of analytical or reagent grade.

\subsection{Preparation and storage of the 2-SeCD stock solution}

Professor Guimin Luo from the Key Laboratory for Molecular Enzymology and Engineering of the Ministry of Education, Jilin University, Changchun, P. R. China kindly provided the 2-SeCD. The 2-SeCD was stored as a solid at room temperature. A $10 \mathrm{mM}$ stock solution was prepared in distilled water, sterilized through a $0.2 \mu \mathrm{m}$ filter and stored at $4{ }^{\circ} \mathrm{C}$.

\subsection{Strain maintenance and nematode culture}

C. elegans Bristol $\mathrm{N}_{2}$ (wild-type) and PD4251 [(pSAK2) myo3p::GFP::LacZ::NLS + (pSAK4) myo-3p::mitochondrial GFP + dpy-20(+)] I strains, both of which were available in our laboratory, were used. The PD4251 strain expressed green fluorescent protein (GFP) in all body wall and vulval muscles, whereas at the cellular level, GFP was expressed in nuclei and mitochondria. Both strains were cultured in plates containing nematode growth medium (NGM; $1.7 \%$ agar and $2.5 \mu \mathrm{g} \mathrm{ml}$ peptone dissolved in $25 \mathrm{mM} \mathrm{KH}_{2} \mathrm{PO}_{4}, 50 \mathrm{mM} \mathrm{NaCl}, 5 \mu \mathrm{g} \mathrm{ml}$ cholesterol, $1 \mathrm{mM} \mathrm{MgSO}_{4}$, and $1 \mathrm{mM} \mathrm{CaCl}_{2}$ ) and the Escherichia coli OP50 strain, which served as a food source, as previously described. ${ }^{15}$ Escherichia coli OP50 is uracil defect and has low metabolic rate, so even if it ate $2-S e C D$ to produce a metabolite, it was negligible comparing with the amount of 2-SeCD applied to the culture medium. Thus, its effect can be ignored for its effect on nematode worms. The 2-SeCD stock solution was diluted by logarithmic phase Escherichia coli OP50 cultured in LB liquid medium to a series concentration. The control group worms were fed with Escherichia coli OP50 only.

\subsection{Lifespan assay}

Twenty gravid adult nematodes were cultured on NGM-OP50 plates (control) or NGM-OP50 plates containing $0.05,0.5$, or $5 \mathrm{mM}$ 2-SeCD and allowed to lay eggs at $20{ }^{\circ} \mathrm{C}$ for approximately $2 \mathrm{~h}$ to obtain a synchronous population. After $2 \mathrm{~h}$, the nematodes were removed, and the plates were placed back at $20{ }^{\circ} \mathrm{C}$ until the progeny reached adulthood $(\sim 72 \mathrm{~h})$. The nematodes were transferred every other day to new NGM-OP50 plates or NGM-OP50 plates containing 2-SeCD. Nematode viability was scored daily or every other day by gentle prodding with a brow. Animals that failed to move were assumed to be dead and not scored, whereas those that exhibited bagging or crawling, or those that exploded upon prodding were scored $(n \geq 40$ per group).

\subsection{Stress resistance assay}

Nematodes were grown on NGM-OP50 plates until a late larval stage (i.e., L4, 96 h after hatching) and subsequently transferred to new NGM-OP50 plates or NGM-OP50 plates containing $0.05,0.5$, or $5 \mathrm{mM} 2$-SeCD for $48 \mathrm{~h}$, with $n \geq 40$ animals per treatment group. Thereafter, nematodes were subjected to heat shock, UV irradiation or $\mathrm{H}_{2} \mathrm{O}_{2}$ oxidation.

2.5.1 Heat shock. The heat shock experiment was carried out according to the method of Lithgow et al. ${ }^{16}$ On the third day of adulthood, nematodes were incubated at $35{ }^{\circ} \mathrm{C}$ and monitored every $1 \mathrm{~h}$ thereafter until all of the animals had died. The control group consisted of nematodes incubated at $20{ }^{\circ} \mathrm{C}$.

2.5.2 UV irradiation. On the third day of adulthood, nematodes were transferred to bacteria-free NGM plates and subjected to UV irradiation $\left(1000 \mathrm{~J} \mathrm{~m}^{-2}\right.$ generated by a single 15 watt UV light bulb) for $8 \mathrm{~min} 40 \mathrm{~s}$. Thereafter, nematodes were transferred to NGM-OP50 plates and monitored daily for survival by gentle prodding with a brow. ${ }^{17}$ The control group consisted of 2-SeCD-untreated nematodes subjected to UV irradiation.

2.5.3 $\mathrm{H}_{2} \mathrm{O}_{2}$ oxidative stress. The $\mathrm{H}_{2} \mathrm{O}_{2}$ oxidative stress experiment was carried out as previously described for the stress resistance assay. On the third day of adulthood, nematodes were transferred to NGM-OP50 plates containing $15 \mathrm{mM}$ $\mathrm{H}_{2} \mathrm{O}_{2}$ and monitored daily for survival. ${ }^{18}$

\subsection{Motility}

Nematode motility can be assessed by two approaches; in the first approach, spontaneous movement is assessed, whereas in the second approach, swimming frequency in liquid culture medium is measured. To assess motility in C. elegans, we used 
the first approach as previously described..$^{19}$ Nematodes with spontaneous and smooth movement, which formed a sinusoidal and symmetric pattern, were regarded as type A, whereas those with limited movement (i.e., only the nose or tail moved upon gentle prodding with a brow) were regarded as type C. Type B was defined as movement falling in between types A and $\mathrm{C}^{20}$ ( $n \geq 40$ per group).

\subsection{Assessment of the body wall muscle integrity}

To assess body wall muscle integrity, the PD4251 strain was used. Newly hatched nematodes were cultured on NGM-OP50 plates containing $5 \mathrm{mM}$ 2-SeCD. The control group consisted of 2-SeCD-untreated nematodes cultured on NGM-OP50 plates. On the fifth day, nematodes were treated with $1 \% \mathrm{NaN}_{3}$ and transferred onto $5 \%$ agarose-coated glass slides. Images were acquired using an inverted fluorescent microscope and intensity was quantified by Image-Pro Plus ( $n \geq 8$ per group).

\subsection{GPX activity}

Nematodes were grown on NGM-OP50 plates from $96 \mathrm{~h}$ after birth to adulthood, and then incubated with $0.05,0.5$, or $5 \mathrm{mM}$ 2-SeCD for $48 \mathrm{~h}$. After $12 \mathrm{~h}$, the nematodes were harvested and washed thrice in $\mathrm{M}_{9}$ buffer $\left(22 \mathrm{mM} \mathrm{KH}_{2} \mathrm{PO}_{4}, 42 \mathrm{mM} \mathrm{Na}_{2} \mathrm{HPO}_{4}\right.$, $85 \mathrm{mM} \mathrm{NaCl}$, and $1 \mathrm{mM} \mathrm{MgSO}_{4}, \mathrm{pH}$ 7.0) to remove residual 2SeCD. ${ }^{21}$ Glutathione peroxidase activity was determined by the method of Wilson et al. ${ }^{22}$ The nematodes were homogenized in $\mathrm{M}_{9}$ buffer, and lysates were centrifuged at $8000 \times g$ for $10 \mathrm{~min}$ at $4{ }^{\circ} \mathrm{C}$. The $500 \mu \mathrm{l}$ reaction contained $50 \mu \mathrm{g}$ of lysate, $50 \mathrm{mM}$ $\mathrm{NaH}_{2} \mathrm{PO}_{4}, 1 \mathrm{mM}$ EDTA, $1 \mathrm{mM} \mathrm{NaN}{ }_{3}, 1 \mathrm{mM}$ glutathione reductase, $0.25 \mathrm{mM}$ NADPH and $1 \mathrm{U}$ glutathione reductase $(\mathrm{pH}$ 7.0), and it was carried out at $37^{\circ} \mathrm{C}$. The reaction was initiated by the addition of $0.5 \mathrm{mM} \mathrm{H}_{2} \mathrm{O}_{2}$. The absorbance of NADPH at $340 \mathrm{~nm}$ corresponded to glutathione peroxidase activity $(n \geq 40$ per group).

\subsection{Lipid peroxidation assay}

Nematodes were divided into three groups, with $n \geq 60$ animals per treatment group as follows: (I-control) newly hatched nematodes were cultured on NGM-OP50 plates only, (II- $\mathrm{H}_{2} \mathrm{O}_{2}$ ) newly hatched nematodes were cultured on NGM-OP50 plates for $144 \mathrm{~h}$, treated with $15 \mathrm{mM} \mathrm{H}_{2} \mathrm{O}_{2}$ for $3 \mathrm{~h}$ in the dark, and then transferred to NGM-OP50 plates, and ( $\mathrm{III}-\mathrm{H}_{2} \mathrm{O}_{2}+2$-SeCD) newly hatched nematodes were cultured on NGM-OP50 plates for $96 \mathrm{~h}$, treated with $5 \mathrm{mM}$ 2-SeCD for $48 \mathrm{~h}$, treated with $15 \mathrm{mM} \mathrm{H}_{2} \mathrm{O}_{2}$ for $3 \mathrm{~h}$ in the dark, and then transferred to NGM-OP50 plates. Nematodes were harvested at $171 \mathrm{~h}$, suspended in $200 \mu \mathrm{l}$ of a mixture of $0.8 \%$ thiobarbituric acid, $16 \%$ trichoroacetic, acid and $8.33 \%$ concentrated sulfuric acid, and then incubated in a water bath set at $95{ }^{\circ} \mathrm{C}$ for $30 \mathrm{~min}$. The absorbance at $532 \mathrm{~nm}$ corresponded to the MDA level ${ }^{23}$ ( $n \geq 40$ per group).

\subsection{Measurement of reactive oxygen species (ROS)}

Intracellular reactive oxygen species (ROS) in C. elegans were determined by dichlorofluorescin diacetate (DCF-DA). Nematodes were collected at $171 \mathrm{~h}$ and treated as described in lipid peroxidation assay. Thereafter, animals were analyzed immediately by a commercially available kit (Beyotime Institute of Biotechnology, China). ${ }^{24-26}$ Samples were read every $20 \mathrm{~min}$ for $2 \mathrm{~h}$ at excitation $488 \mathrm{~nm}$ and emission $525 \mathrm{~nm}$.

\subsection{Determination of the brood size}

After birth, the nematodes were individually grown on NGMOP50 plates in the absence (control) or presence of $5 \mathrm{mM} \mathrm{2-}$ SeCD, with 10 animals per treatment group. Thereafter, animals were transferred every $12 \mathrm{~h}$ to new NGM-OP50 plates until egg production had ceased. The total number of progeny for each treatment group was calculated ${ }^{27}$ ( $n \geq 8$ per group).

\subsection{Statistical analysis}

Statistical analysis was performed using Prism 4 software (GraphPad Software, Inc., La Jolla, CA, USA). Results were expressed as the mean \pm standard deviation of three or four independent experiments. The lifespan of nematodes cultured in the absence or presence of 2-SeCD was compared between groups using the two-tailed, unpaired Student's $t$-test. $P$-values $<$ 0.05 were taken as statistically significant $\left(0.01 \leq{ }^{*} p<0.05\right.$, $\left.0.001 \leq{ }^{* *} p<0.01,{ }^{* * *} p<0.001\right)$.

\section{Results}

\subsection{2-SeCD increases the lifespan of C. elegans}

The reported mean lifespan of wild-type C. elegans nematodes is 2-3 weeks at $20^{\circ} \mathrm{C}$, which is consistent with our results $(16.58 \pm$ 2.11 days, with a maximum of 21 days). After the treatment of newly hatched nematodes with $0.05,0.5$, or $5 \mathrm{mM} 2$-SeCD, the mean lifespan was $17.06 \pm 2.18$ days (with a maximum of 21 days), $17.78 \pm 2.70$ days (with a maximum of 22 days), and 18.26

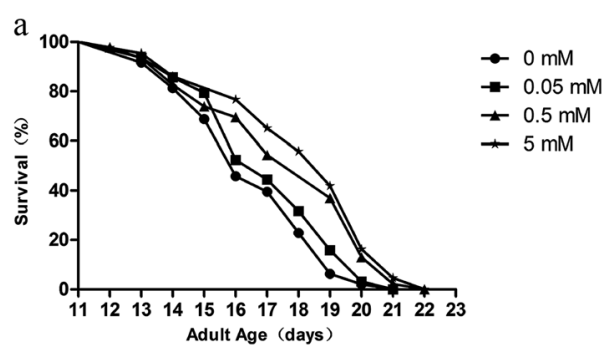

b

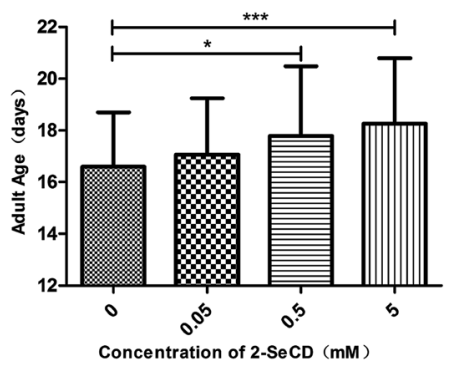

Fig. 1 Effect of different concentrations of 2-SeCD on wild type C. elegans lifespan from born at $20^{\circ} \mathrm{C}$. Animals showed longevity consist with control in a dose-dependent manner. ( $N \geq 40$ per group, $0.01 \leq$ $* p<0.05,0.001 \leq * * p<0.01, * * * p<0.001)$. 


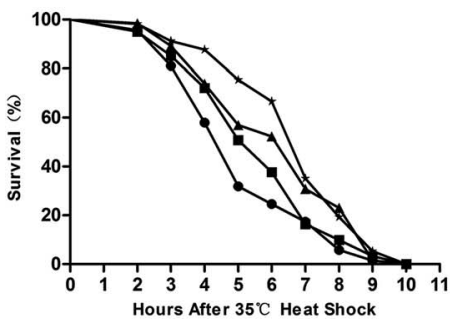

a

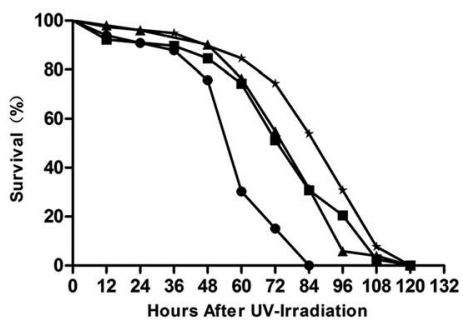

c

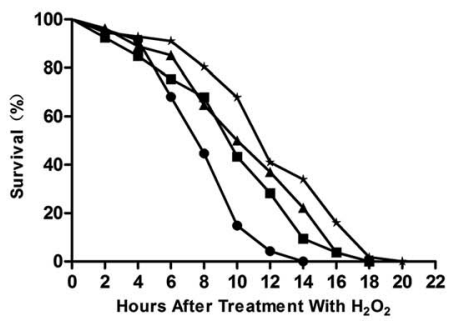

e

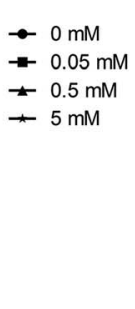

$\rightarrow 0 \mathrm{mM}$

$-0.05 \mathrm{mM}$

$+0.5 \mathrm{mM}$

- $5 \mathrm{mM}$

$-0 \mathrm{mM}$

$-0.05 \mathrm{mM}$

$+0.5 \mathrm{mM}$

$+5 \mathrm{mM}$

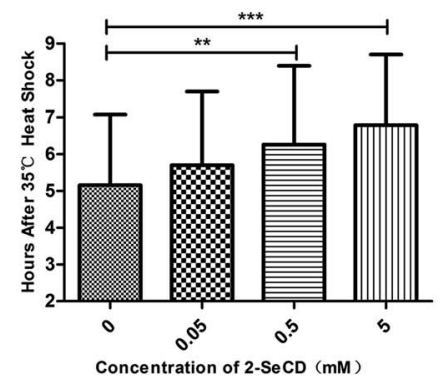

b

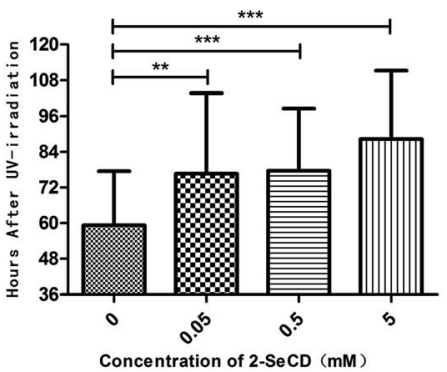

d

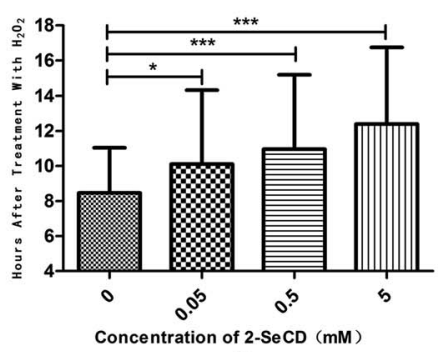

$\mathrm{f}$

Fig. 2 Effect of pretreatment with $2-S e C D$ on resistance to stress in C. elegans. ( $N \geq 40$ per group, $0.01 \leq * p<0.05,0.001 \leq * * p<0.01, * * * p<$ 0.001). Animals were treated with a series concentration of $2-\mathrm{SeCD}$ from born for 120 hours at $20^{\circ} \mathrm{C}$ and exposed to a variety of stressors. Animals treated with 2-SeCD had more resistance to stress than control. Each experiment is representative of three independent trials. (a, b) $35^{\circ} \mathrm{C}$ heat shock. (c, d) UV irradiation at $1000 \mathrm{~J} \mathrm{~m}^{-2}$. (e, f) $15 \mathrm{mM} \mathrm{H}_{2} \mathrm{O}_{2}$.

\pm 2.54 days (with a maximum of 22 days), respectively, indicating that 2-SeCD could significantly affect the lifespan of $C$. elegans (Fig. 1a and b).

\subsection{2-SeCD increases the resistance of C. elegans to stress}

Young adult nematodes were treated with $0.05,0.5$, or $5 \mathrm{mM} 2-$ SeCD for $48 \mathrm{~h}$. Thereafter, their responses to a variety of physical and chemical stressors were assessed.

3.2.1 Heat shock. To induce stress by heat shock, nematodes were incubated in the absence (control) or presence of 2SeCD for $48 \mathrm{~h}$ and then incubated at $35{ }^{\circ} \mathrm{C}$ until all of the animals had died. Although $69 \%$ of control nematodes died after $5 \mathrm{~h}$ of heat shock, $51 \%, 57 \%$, and $75 \%$ of nematodes were viable after treatment with $0.05,0.5$, and $5 \mathrm{mM} 2$-SeCD, respectively (Fig. 2a and b).

3.2.2 UV irradiation. To induce stress by UV irradiation, nematodes were incubated in the absence (control) or presence of 2-SeCD and then exposed to UV irradiation $\left(1000 \mathrm{~J} \mathrm{~m}^{-2}\right.$ generated by a single 15 watt UV light bulb). Nematodes treated with 2-SeCD survived significantly longer than control nematodes (Fig. 2c and d).
3.2.3 $\mathrm{H}_{2} \mathrm{O}_{2}$ oxidation. To induce stress by $\mathrm{H}_{2} \mathrm{O}_{2}$ oxidation, nematodes were incubated in the absence (control) or presence of 2-SeCD for $48 \mathrm{~h}$, and then cultured on NGM-OP50 plates containing $15 \mathrm{mM} \mathrm{H}_{2} \mathrm{O}_{2}$. Although control nematodes survived for an average of $8.47 \mathrm{~h}$, those treated with $0.05,0.5$, or $5 \mathrm{mM}$ 2-SeCD survived for an average of $10.11 \mathrm{~h}$ (with a maximum of $18 \mathrm{~h}$ ), $10.97 \mathrm{~h}$ (with a maximum of $18 \mathrm{~h}$ ), and $12.39 \mathrm{~h}$ (with a maximum of $20 \mathrm{~h}$ ), respectively, representing increases of 19\%, 29\%, and 46\% over control nematodes (Fig. 2e and f).

\subsection{2-SeCD improves nematode motility}

We investigated the motility of 10-, 12- and 14 day-old nematodes treated with 2-SeCD. There was a significant dosedependent decline in the motility of 10-, 12- and 14 day-old nematodes. Whether animals treated with 2-SeCD or not, most 10 day-old nematodes moved spontaneously, and there a marked difference in spontaneous motility (type A) between the control and treatment groups. Furthermore, 12 day-old nematodes treated with $0.05,0.5$ and $5 \mathrm{mM}$ 2-SeCD showed increased spontaneous motility $(33 \%, 46 \%$ and $62 \%$, respectively) compared with that of the control group (8\%). The most 


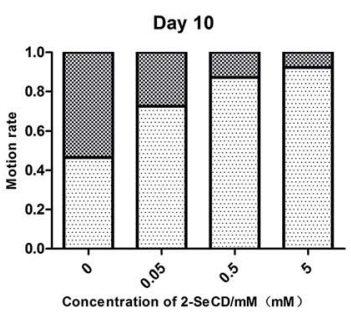

a

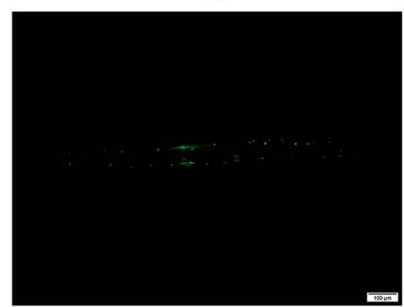

e

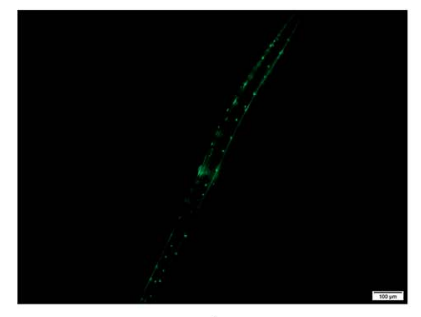

Day 12

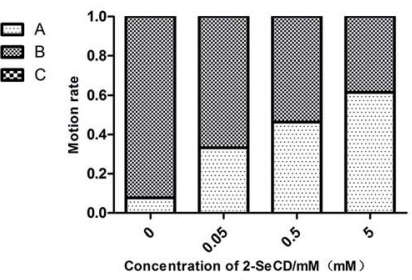

b

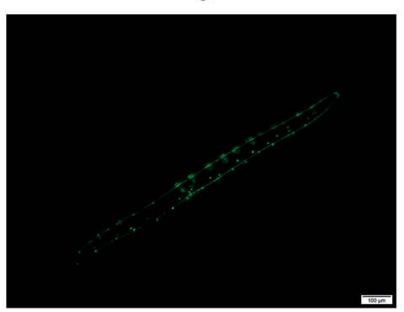

f

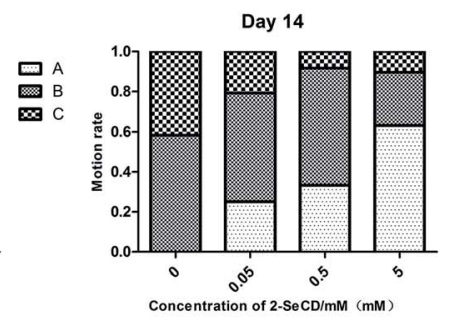

C

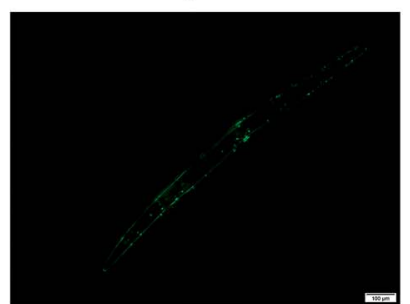

g

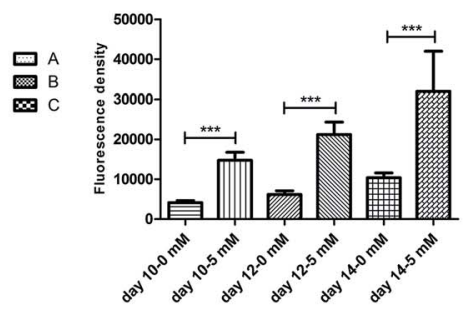

d

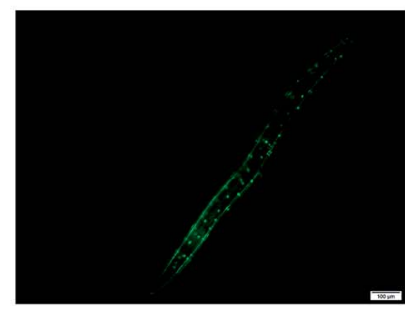

h

i

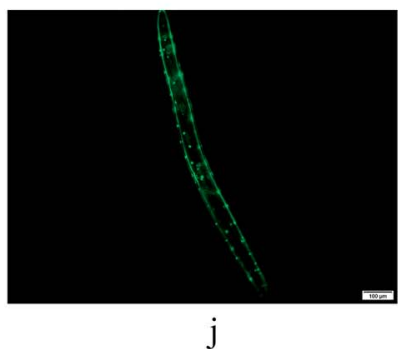

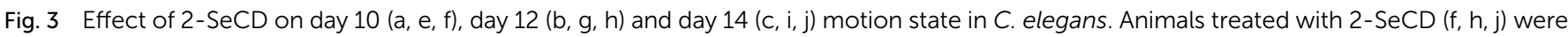
more active than control $(\mathrm{e}, \mathrm{g}, \mathrm{i})$ in a dose-dependent manner (d) $(N>40$ per group, $0.01<* p<0.05,0.001<* * p<0.01, * * * p<0.001)$.

apparent changes occurred in 14 day-old nematodes; $42 \%$ of nematodes in the control group barely moved their heads after gently prodding with a brow (type C) compared to the treatment groups (less than $20 \%$ ), whereas $25 \%$ of the nematodes treated with $5 \mathrm{mM}$ 2-SeCD showed spontaneous movement (Fig. 3a-c). To determine if 2-SeCD improves muscle function, we treated the PD4251 strain with $5 \mathrm{mM}$ 2-SeCD and found an increased number of GFP light spots and bright points on the body wall compared to the control, suggesting that 2-SeCD enhances motility by strengthening body wall muscles (Fig. 3d-j).

\subsection{2-SeCD increases glutathione peroxidase activity in nematode}

We investigated whether 2-SeCD can increase glutathione peroxidase activity and resistance to ROS generators. We found that 2-SeCD increased the glutathione peroxidase activity level. 2-SeCD at $0.05 \mathrm{mM}$ increased total glutathione peroxidase activity levels in whole homogenates from treated worms in the manner of dose-dependence (Fig. 4).

\subsection{2-SeCD decreases the lipid peroxidation and ROS level in nematode}

The lipid peroxidation product, MDA, is often used to measure lipid peroxidation. We found that the MDA level was higher in $\mathrm{H}_{2} \mathrm{O}_{2}$-treated nematodes than in control nematodes. Due to the effects of 2-SeCD on nematodes, the effect of the highest concentration of 2-SeCD on the MDA level was also tested and $5 \mathrm{mM}$ 2-SeCD was used for this purpose. Before the onset of $\mathrm{H}_{2} \mathrm{O}_{2}$-induced damage but after the treatment with $5 \mathrm{mM}$ 2-SeCD for $48 \mathrm{~h}$, the MDA level declined, indicating that 2-SeCD inhibits lipid peroxidation (Fig. 5a). Moreover, ROS level in the wild type nematodes fed with $5 \mathrm{mM}$ 2-SeCD was shown in Fig. 5b. Significant decline were observed for $5 \mathrm{mM}$ 2-SeCD treatments compared with control, indicating that 2-SeCD could also decrease ROS level in C. elegans (Fig. 5b).

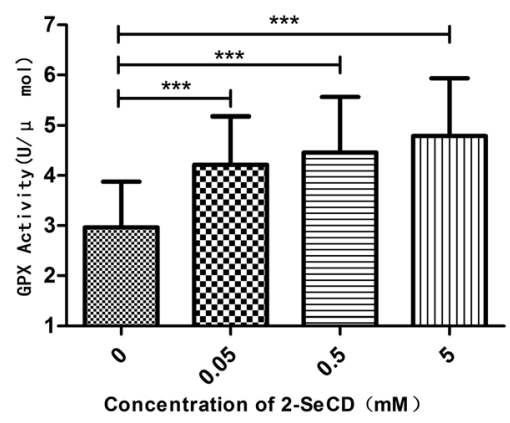

Fig. 4 Effect of 2-SeCD on GPX activity in C. elegans. Animals treated with 2-SeCD had higher GPX activity than control in a dose-dependent manner. ( $n>40$ per group, $0.01<* p<0.05,0.001<* * p<0.01, * * * p<$ 0.001). 


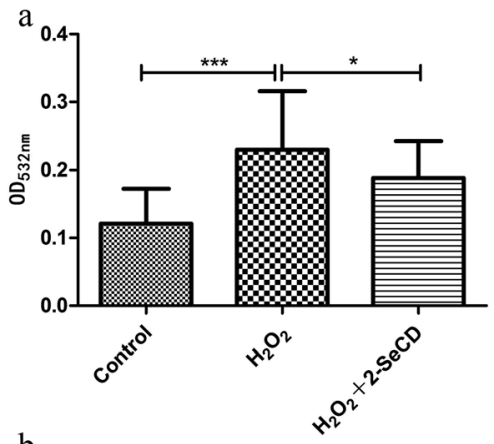

b

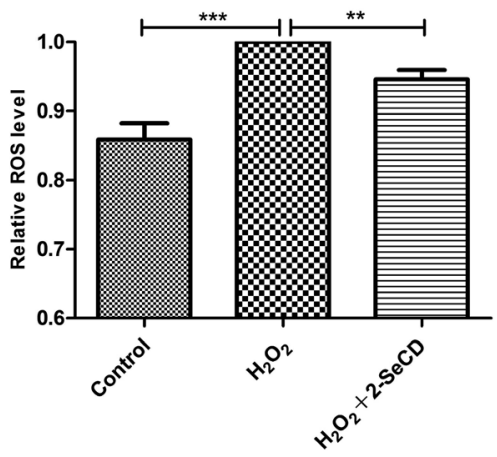

Fig. 5 Effect of 2-SeCD on lipid peroxidation and ROS level in C. elegans under $15 \mathrm{mM} \mathrm{H}_{2} \mathrm{O}_{2}$ condition. 2-SeCD could effectively reduced the damage by $\mathrm{H}_{2} \mathrm{O}_{2}$ in nematodes ( $n>40$ per group. $0.01<$ $* p<0.05,0.001<* * p<0.01, * * * p<0.001)$.

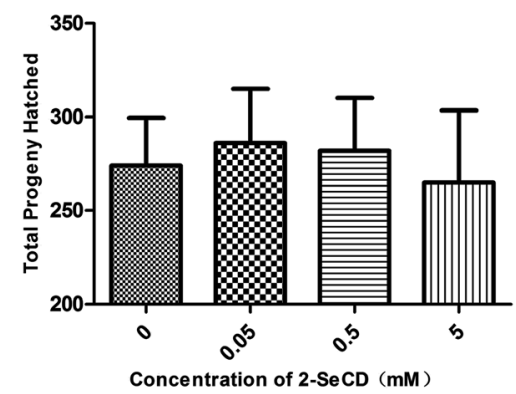

Fig. 6 Effect of arbutin on brood size of C. elegans. There was no significant difference in the number of total progeny between 2SeCD-treated and untreated animals. ( $n>8$ per group, $0.01<* p<$ $0.05,0.001<* * p<0.01, * * * p<0.001)$

\section{6-SeCD does not affect the nematode brood size}

To determine whether 2-SeCD affects fecundity, we measured the brood size of nematodes cultured in the absence (control) or presence of $0.05,0.5$, or $5 \mathrm{mM}$ 2-SeCD. There was no significant difference in the brood size between the control and treatment groups (Fig. 6).

\section{Discussion}

Several studies have shown that 2-SeCD has glutathione peroxidase-like activity. In this study, we examined the effects of 2-SeCD on several key parameters in C. elegans. We found that 2SeCD was non-toxic at the concentrations used in this study.
Our key findings were that dietary 2-SeCD enhanced the lifespan of nematodes, increased their resistance to environmental stress in a dose-dependent manner, enhanced their motility, as well as increased GPX activity and decreased lipid peroxidation and ROS level.

We first investigated the effect of 2-SeCD on worms and found it could extend lifespan and have no toxicity to animals in suitable dose. Then we measured the brood size of animals treated with 2-SeCD and found that there was no difference between the groups, indicating that 2-SeCD was non-toxic to $C$. elegans. Next, we investigated whether 2-SeCD had similar effects in nematodes as those in other species. For the lifespan and brood size assays, worms were treated with 2-SeCD since eggs, and for the stress resistance assay since L4. It was because that lifespan and brood size assays were to reflect the effects of 2-SeCD on development and reproduction, however, it was necessary to ensure animals health statement before stresses. The results were all positive. We found that 2-SeCD not only improved resistance to heat shock, UV irradiation and oxidation induced by $\mathrm{H}_{2} \mathrm{O}_{2}$, but also extended the lifespan and benefited the healthspan of nematodes, especially their motility. We also used the PD4251 strain to investigate muscle integrity and agedependent muscle degeneration or sarcopenia. The transgenic C. elegans strain (PD4251) expressing green fluorescent protein (GFP)-MYO-3, localized in body wall muscles and vulval muscle nuclei, was fed with 2-SeCD, and muscle integrity was analyzed by quantification of GFP fluorescence. ${ }^{28,29}$ Animals treated with 2-SeCD expressed more GFP than the control.

Moreover, to investigate the effects of 2-SeCD on oxidation in nematodes, we measured GPX activity, lipid peroxidation level and ROS level. 2-SeCD improved GPX activity and reduced the MDA level, which was consistent with our earlier results and may explain why 2-SeCD improved the lifespan and healthspan of C. elegans. Oxidations in lipid peroxidation level and ROS level assay were all induced by $15 \mathrm{mM} \mathrm{H}_{2} \mathrm{O}_{2}$. Glutathione peroxidase is one of the most important enzymes in aerobic organisms, and it can scavenge free radicals and hydrogen peroxide. ${ }^{30}$ Selenium, in the form of selenocysteine, is a necessary component of GPX activity. With aging, nematodes became slow moving and finally inactive. After the treatment with various stressors, nematodes quickly aged or died. However, nematodes treated with 2-SeCD exhibited a longer lifespan, more rigorous movement and stronger resistance to stress than untreated nematodes. Reactive oxygen species degrade polyunsaturated lipids, forming malondialdehyde. This compound is a reactive aldehyde and is one of the many reactive electrophile species that cause toxic stress in cells. The production of this aldehyde is used as a biomarker to measure the level of oxidative stress in an organism. Under a normal physiological state, the body content of MDA is very low, and its content can reflect the level of body cells which are attacked by free radicals. ${ }^{31}$ It has been reported that 2 -SeCD has widespread effects in other models. For example, Mu and coworkers showed that 2SeCD protects keratinocytes from apoptosis, inhibits lipid peroxidation, and scavenges ROS. ${ }^{32}$ Likewise, Sun and colleagues showed that 2-SeCD decreased UVB irradiation-induced p53 and Bcl-2 levels. ${ }^{33}$ Another research group demonstrated that 2- 
SeCD might be useful during liver surgery. ${ }^{34}$ C. elegans represents a useful model in aging research because nematodes decline behaviorally and physiologically with age in a manner similar to that of higher mammals, including humans. ${ }^{35}$ It is also a widespread model used to study human pharmacological interventions, ranging from vitamins and clinical drugs to antioxidant supplements and phytochemicals. ${ }^{36}$ The results showed that 2-SeCD enhanced GPX activity and reduced lipid peroxidation and ROS levels.

It is known that specific human pharmacological interventions, including vitamins, clinical drugs, antioxidant supplements and phytochemicals, can extend the lifespan or delay physiological aging in C. elegans. ${ }^{37}$ For example, extracts of Ginkgo biloba, ${ }^{38}$ and components of blueberry polyphenols, ${ }^{39}$ extend the lifespan and healthspan of nematodes, and enhance stress resistance through a variety of mechanisms. Despite these and other studies, however, surprisingly little is known about the possible anti-aging effects of 2-SeCD in vivo, and this was precisely our study goal. As for the mechanism of action of 2-SeCD, we speculate that it is involved in the signaling pathways associated with aging. Further studies are needed to address the mechanism of action. Moreover, we intend to explore the effects of 2-SeCD in higher animals, which should strengthen the foundation of this GPX-like compound in research and development.

\section{Conclusions}

In summary, we demonstrated that 2-SeCD, which was nontoxic at the concentrations used in this study, extended the lifespan of C. elegans. Nematodes treated with 2-SeCD were more resistant to heat, UV irradiation, and $\mathrm{H}_{2} \mathrm{O}_{2}$-induced oxidative stress, indicating that cellular defenses and immunological parameters were improved. In addition, results from experiments that measured motility, GPX activity and the MDA level revealed that 2-SeCD improved the healthspan of C. elegans. Further studies are needed to elucidate the mechanisms behind these health benefits.

\section{Conflicts of interest}

There are no conflicts to declare.

\section{Acknowledgements}

This work was supported by grants from the National Natural Science Foundation of China (No. 31000358 for Junfeng Ma and No. 31670795 for Xueqi Fu), Jilin Province Postdoctoral Science Foundation (for Junfeng Ma) and Changbai Mountain Research Support Foundation of 2017 (No. 440050117010 for Xueqi Fu). Especially, we thank Professor Guimin Luo from the Key Laboratory for Molecular Enzymology and Engineering of the Ministry of Education, Jilin University, Changchun, P. R. China for providing 2-SeCD and assisting with the enzyme experiment. Moreover, C. elegans strains were provided by the CGC, which funded by NIH Office of Research Infrastructure Programs (P40 OD010440).

\section{References}

1 D. Harman, J. Gerontol., 1956, 11, 298-300.

2 R. S. Sohal, Aging (Milano, Italy), 1993, 5, 3-17.

3 T. Finkel and N. J. Holbrook, Nature, 2000, 408, 239-247.

4 G. M. Martin, S. N. Austad and T. E. Johnson, Nat. Genet., 1996, 13, 25-34.

5 H. Adachi, Y. Fujiwara and N. Ishii, J. Gerontol., Ser. A, 1998, 53, B240-B244.

6 M. Keaney, F. Matthijssens, M. Sharpe, J. Vanfleteren and D. Gems, Free Radical Biol. Med., 2004, 37, 239-250.

7 M. J. Corey and E. Corey, Proc. Natl. Acad. Sci. U. S. A., 1996, 93, 11428-11434.

8 Z. Wu, J. V. Smith, V. Paramasivam, P. Butko, I. Khan, J. R. Cypser and Y. Luo, Cell. Mol. Biol., 2002, 48, 725-731.

9 J. J. Collins, K. Evason and K. Kornfeld, Exp. Gerontol., 2006, 41, 1032-1039.

10 X. F. Zou, Y. T. Ji, G. Gao, X. J. Zhu, S. W. Lv, F. Yan, S. P. Han, X. Chen, C. C. Gao, J. Liu and G. M. Luo, J. Microbiol. Biotechnol., 2010, 20, 88-93.

11 J. Liu, G. Luo, X. Ren, Y. Mu, Y. Bai and J. Shen, Biochim. Biophys. Acta, Biomembr., 2000, 1481, 222-228.

12 T. Maraldi, C. Prata, D. Fiorentini, L. Zambonin, L. Landi and G. Hakim, Free Radical Biol. Med., 2009, 46, 244-252.

13 T. E. Johnson, Exp. Gerontol., 2003, 38, 1329-1332.

14 R. Bell, A. Hubbard, R. Chettier, D. Chen, J. P. Miller, P. Kapahi, M. Tarnopolsky, S. Sahasrabuhde, S. Melov and R. E. Hughes, PLoS Genet., 2009, 5, e1000414.

15 H. D. Mistry, V. Wilson, M. M. Ramsay, M. E. Symonds and F. Broughton Pipkin, Hypertension, 2008, 52, 881-888.

16 G. J. Lithgow, T. M. White, S. Melov and T. E. Johnson, Proc. Natl. Acad. Sci. U. S. A., 1995, 92, 7540-7544.

17 J. R. Cypser and T. E. Johnson, J. Gerontol., Ser. A, 2002, 57, B109-B114.

18 M. W. Tan, S. Mahajan-Miklos and F. M. Ausubel, Proc. Natl. Acad. Sci. U. S. A., 1999, 96, 715-720.

19 T. R. Golden, A. Hubbard, C. Dando, M. A. Herren and S. Melov, Aging Cell, 2008, 7, 850-865.

20 L. A. Herndon, P. J. Schmeissner, J. M. Dudaronek, P. A. Brown, K. M. Listner, Y. Sakano, M. C. Paupard, D. H. Hall and M. Driscoll, Nature, 2002, 419, 808-814.

21 E. Possik and A. Pause, J. Visualized Exp., 2015, e52746, DOI: $10.3791 / 52746$.

22 S. R. Wilson, P. A. Zucker, R. R. C. Huang and A. Spector, J. Am. Chem. Soc., 1989, 111, 5936-5939.

23 Y. Sun, Y. Mu, W. Li, S. Lv, Z. Jiang, K. Zhang, K. Zheng, F. Lin, G. Yan, G. Luo, J. Liu and J. Shen, Arch. Biochem. Biophys., 2003, 412, 90-94.

24 L. Zhang, G. Jie, J. Zhang and B. Zhao, Free Radical Biol. Med., 2009, 46, 414-421.

25 S. Abbas and M. Wink, Planta Med., 2009, 75, 216-221.

26 M. K. Brown, J. L. Evans and Y. Luo, Pharmacol., Biochem. Behav., 2006, 85, 620-628.

27 J. Li, A. Ebata, Y. Dong, G. Rizki, T. Iwata and S. S. Lee, PLoS Biol., 2008, 6, e233. 
28 Z. Cao, Y. Wu, K. Curry, Z. Wu, Y. Christen and Y. Luo, J. Gerontol., Ser. A, 2007, 62, 1337-1345.

29 Q. Yan, R. Zhao, W. Zheng, C. Yin, B. Zhang and W. Ma, BMC Biotechnol., 2009, 9, 47.

30 J. F. Atkins and R. F. Gesteland, Nature, 2000, 407, 463-465.

31 E. Papadimitriou and N. S. Loumbourdis, Bull. Environ. Contam. Toxicol., 2002, 69, 885-891.

32 Y. Mu, S. Lv, X. Ren, G. Jin, J. Liu, G. Yan, W. Li, J. Shen and G. Luo, J. Photochem. Photobiol., B, 2003, 69, 7-12.

33 Y. Sun, Y. Mu, S. Ma, P. Gong, G. Yan, J. Liu, J. Shen and G. Luo, Biochim. Biophys. Acta, Biomembr., 2005, 1743, 199204.
34 T. T. Lin, B. M. Wang, X. Y. Li, Y. Pan, W. Wang, Y. Mu, J. Q. Liu, J. C. Shen and G. M. Luo, Hepatol. Res., 2009, 39, 1125-1136.

35 M. Lucanic, G. J. Lithgow and S. Alavez, Ageing Res. Rev., 2013, 12, 445-458.

36 E. M. Vayndorf, S. S. Lee and R. H. Liu, J. Funct. Foods, 2013, 5, 1236-1243.

37 M. Petrascheck, X. Ye and L. B. Buck, Ann. N. Y. Acad. Sci., 2009, 1170, 698-701.

38 P. Y. Zhang, X. Xu and X. C. Li, Eur. Rev. Med. Pharmacol. Sci., 2014, 18, 3091-3096.

39 M. A. Wilson, B. Shukitt-Hale, W. Kalt, D. K. Ingram, J. A. Joseph and C. A. Wolkow, Aging Cell, 2006, 5, 59-68. 